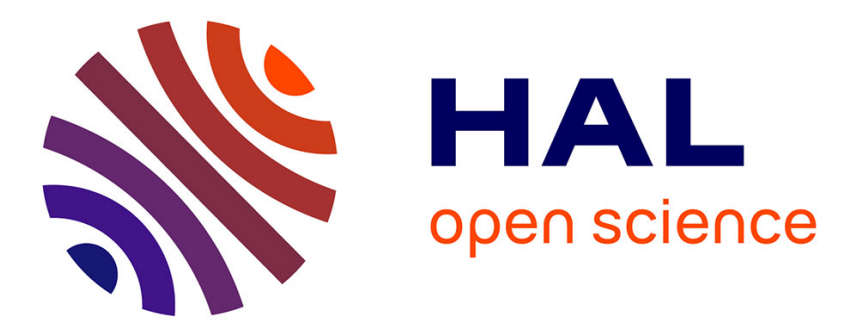

\title{
Finite tunnel magnetoresistance at the compensation point of Sm1xGdxAl2, a ferromagnetic electrode with zero magnetization Related Articles
}

M da Silva, K Dumesnil, C Dufour, M Hehn, D Pierre, D Lacour, F. Montaigne, G. Lengaigne, S. Robert

\section{To cite this version:}

M da Silva, K Dumesnil, C Dufour, M Hehn, D Pierre, et al.. Finite tunnel magnetoresistance at the compensation point of Sm1xGdxAl2, a ferromagnetic electrode with zero magnetization Related Articles. Applied Physics Letters, 2011, 98 (23), pp.232504. 10.1063/1.3597625 . hal-02949054

\section{HAL Id: hal-02949054 https://hal.science/hal-02949054}

Submitted on 25 Sep 2020

HAL is a multi-disciplinary open access archive for the deposit and dissemination of scientific research documents, whether they are published or not. The documents may come from teaching and research institutions in France or abroad, or from public or private research centers.
L'archive ouverte pluridisciplinaire HAL, est destinée au dépôt et à la diffusion de documents scientifiques de niveau recherche, publiés ou non, émanant des établissements d'enseignement et de recherche français ou étrangers, des laboratoires publics ou privés. 


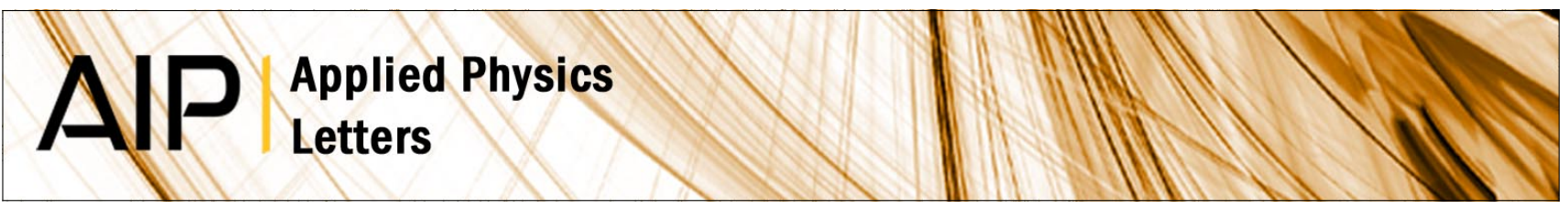

\section{Finite tunnel magnetoresistance at the compensation point of Sm1xGdxAl2, a ferromagnetic electrode with zero magnetization}

M. Da Silva, K. Dumesnil, C. Dufour, M. Hehn, D. Pierre et al.

Citation: Appl. Phys. Lett. 98, 232504 (2011); doi: 10.1063/1.3597625

View online: http://dx.doi.org/10.1063/1.3597625

View Table of Contents: http://apl.aip.org/resource/1/APPLAB/v98/i23

Published by the American Institute of Physics.

\section{Related Articles}

Barrier height and tunneling aspects in (110) $\mathrm{CrO} 2$ with its natural barrier J. Appl. Phys. 110, 053708 (2011)

Rapid thermal annealing study of magnetoresistance and perpendicular anisotropy in magnetic tunnel junctions based on $\mathrm{MgO}$ and CoFeB

Appl. Phys. Lett. 99, 102502 (2011)

Inductive determination of the optimum tunnel barrier thickness in magnetic tunneling junction stacks for spin torque memory applications

J. Appl. Phys. 110, 023906 (2011)

Effect of metallic Mg insertion on the magnetoresistance effect in MgO-based tunnel junctions using D022-Mn3Ga perpendicularly magnetized spin polarizer

J. Appl. Phys. 110, 013915 (2011)

The perpendicular anisotropy of Co40Fe40B20 sandwiched between Ta and MgO layers and its application in $\mathrm{CoFeB} / \mathrm{MgO} / \mathrm{CoFeB}$ tunnel junction

Appl. Phys. Lett. 99, 012502 (2011)

\section{Additional information on Appl. Phys. Lett.}

Journal Homepage: http://apl.aip.org/

Journal Information: http://apl.aip.org/about/about_the_journal

Top downloads: http://apl.aip.org/features/most_downloaded

Information for Authors: http://apl.aip.org/authors

\section{ADVERTISEMENT}

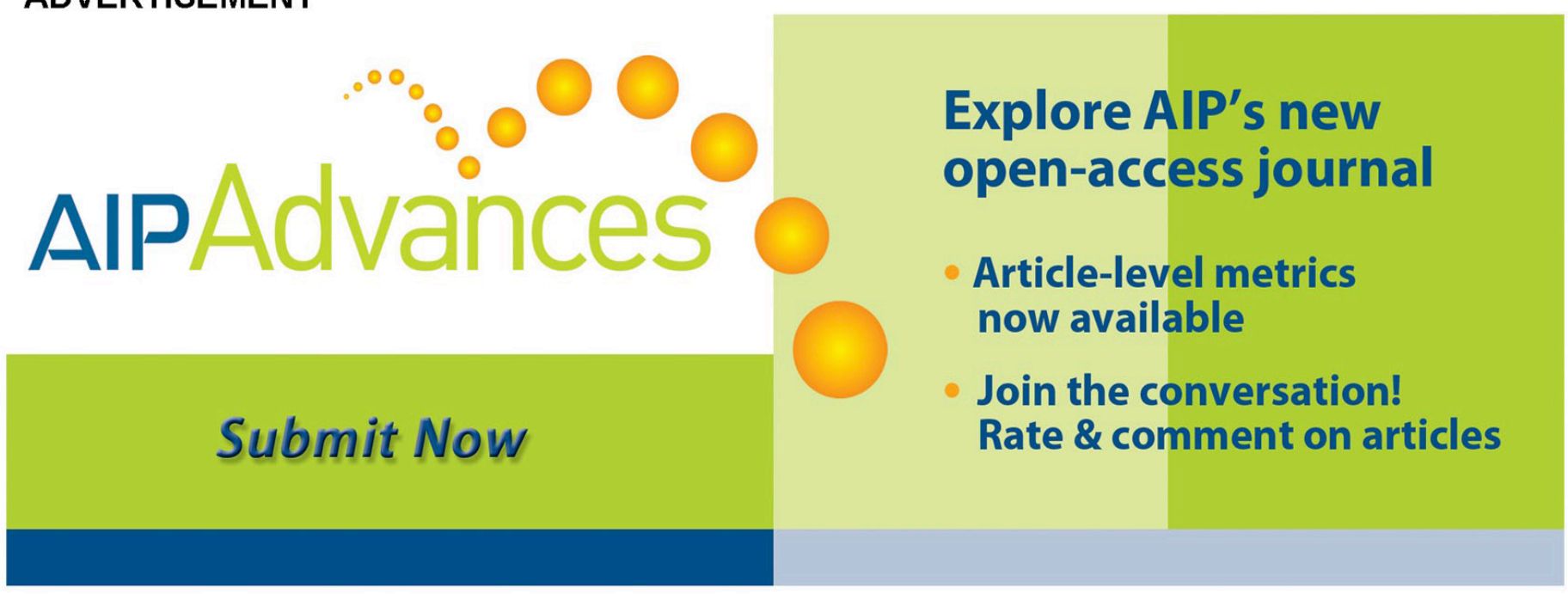




\title{
Finite tunnel magnetoresistance at the compensation point of $\mathrm{Sm}_{1-\mathrm{x}} \mathrm{Gd}_{\mathrm{x}} \mathbf{A l}$, a ferromagnetic electrode with zero magnetization
}

\author{
M. Da Silva, K. Dumesnil, ${ }^{\text {a) }}$ C. Dufour, M. Hehn, D. Pierre, D. Lacour, F. Montaigne, \\ G. Lengaigne, and S. Robert \\ Institut Jean Lamour, UMR CNRS 7198, Nancy-Université, BP 239, F-54506 Vandoeuvre lès Nancy, France
}

(Received 22 March 2011; accepted 15 May 2011; published online 6 June 2011)

\begin{abstract}
A tunnel magnetoresistance effect is observed in magnetic tunnel junctions with an original zero magnetization ferromagnetic electrode composed of $\mathrm{Sm}_{1-\mathrm{x}} \mathrm{Gd}_{\mathrm{x}} \mathrm{Al}_{2}$. A finite tunnel magnetoresistance is measured when the $\mathrm{Sm}_{1-\mathrm{x}} \mathrm{Gd}_{\mathrm{x}} \mathrm{Al}_{2}$ electrode is in a magnetic compensated state proving that $\mathrm{Sm}_{1-\mathrm{x}} \mathrm{Gd}_{\mathrm{x}} \mathrm{Al}_{2}$ with zero magnetization is able to give rise to a spin-polarized current. The temperature dependence of tunnel magnetoresistance reveals that this effect is related to the relative orientation of spin contributions to magnetic moment in both electrodes and that the tunnel polarization of the $\mathrm{Sm}_{1-\mathrm{x}} \mathrm{Gd}_{\mathrm{x}} \mathrm{Al}_{2}$ electrode is negative. (C) 2011 American Institute of Physics. [doi:10.1063/1.3597625]
\end{abstract}

Since the discovery of spin dependent tunneling (SDT) across a Ge barrier by Jullière, ${ }^{1}$ SDT has been shown to occur with the use of other tunnel barrier materials like $\mathrm{Al}_{2} \mathrm{O}_{3}, \mathrm{NiO}, \mathrm{MgO}, \mathrm{HfO}, \mathrm{SrTiO}$, and $\mathrm{TaO} .^{2}$ However, in those reported results, the electrodes were made mainly based on conventional magnetic $3 d$ transition metals (TMs). The next challenge consists in developing electrodes with materials of specific structural and/or magnetic configurations (epitaxial materials, ${ }^{3}$ materials with magnetization perpendicular to the film plane ${ }^{4}$ ), or with materials such as Heusler compounds with predicted half-metallicity. ${ }^{5}$ An intense research activity also aims at designing materials with exciting properties that would permit to get a perfectly stable spin-polarized electrode in a junction device. An ideal compound would be an half-metallic antiferromagnet ${ }^{6}$ or ferrimagnet, ${ }^{7}$ exhibiting both $100 \%$ spin polarization and very small or zero magnetization. Such materials would be very useful for device applications because they would eliminate magnetic dipole fields that can give rise to significant magnetic coupling within and between devices and they would be insensitive to applied field. Up to now, they remain nevertheless only theoretical predictions and their experimental synthesis obviously requires further effort.

Classical ferrimagnetic rare earth (RE)/TM alloys have been already used as electrode in magnetic tunnel junctions (MTJs) and give rise to interesting properties. ${ }^{8}$ Especially at the compensation point of the Gd-Co alloy, where the subnetwork magnetizations are equal and the alloy has nearly zero net magnetization, tunneling spin polarization is maintained as deduced from the observed finite tunnel magnetoresistance (TMR). This can be understood by considering the relative contributions of independent spin-polarized tunneling currents from the $\mathrm{Gd}$ and the transition Co subnetwork magnetizations. Although these RE/TM ferrimagnets can give rise to spin-polarized current in their magnetic compensated state, the zero magnetization state results from opposite spin orders. Beyond RE/TM ferrimagnets, interesting and original materials are developed to achieve simultaneously a zero magnetization state and long range ferromagnetic order where all spin contributions point in the same direction.

${ }^{\text {a)}}$ Electronic mail: dumesnil@1pm.u-nancy.fr.
These are Sm-based zero magnetization ferromagnets (ZMFs) that can also be considered as "self-ferrimagnets.", In these specific compounds, the magnetic compensation does not result from opposite moments located on two different magnetic sublattices, as it is the case in ferrimagnets, but from opposite magnetic contributions located on a single magnetic sublattice. In Sm-based ferromagnets as $\mathrm{SmAl}_{2}$, the magnetic moments, namely, originate from opposite spin (S) and orbital (L) contributions that almost cancel each other. The substitution of a small amount of Sm atoms with $\mathrm{Gd}$ atoms that bring supplemental pure $\mathrm{S}$ contribution leads to a magnetic compensation point $(\mathrm{M}=0)$ at the so-called compensation temperature $\left(\mathrm{T}_{\text {comp }}\right)$, where the $\mathrm{S}$ and $\mathrm{L}$ moments contributions perfectly cancel each other. L moments are the dominant contribution to magnetization below $\mathrm{T}_{\text {comp }}$ while $\mathrm{S}$ moments are dominant above $\mathrm{T}_{\text {comp }}{ }^{10}$ The nature of this unusual compensated state in $\mathrm{Sm}_{1-x} \mathrm{Gd}_{\mathrm{x}} \mathrm{Al}_{2}$ (SGA) results in a $\mathrm{ZM}$ long range ferromagnetic order of $\mathrm{S}$ moments that could generate a spin-polarized current. ${ }^{11,12}$ Our group has proved the possibility to grow this material as epitaxial films of high crystal quality. ${ }^{13}$ We showed that epitaxial films have interesting properties required for fundamental studies in the field of spin-resolved devices: they do exhibit a magnetic compensated state that is directly related to the Gd content, ${ }^{14}$ and this compensated state coexists with a long range ferromagnetic order. ${ }^{15}$

The study presented in this letter deals with the use of this magnetic material (the ZMF SGA), as an original electrode in MTJs. The [111] SGA $(x=2.5 \%)$ layer was epitaxially grown by molecular beam epitaxy (MBE) at $500{ }^{\circ} \mathrm{C}$ in a chamber with base pressures around $10^{-10}$ Torr. A $50 \mathrm{~nm} \mathrm{Nb}$ buffer layer was first deposited on the (11-20) $\mathrm{Al}_{2} \mathrm{O}_{3}$ substrate in order to promote the epitaxial growth of [111] SGA. ${ }^{13}$ Before film deposition, the Sm, Al, and Gd evaporation rates were carefully calibrated in order to get the desired stoichiometry. The layer thickness was $1 \mu \mathrm{m}$. The crystalline quality was checked during growth by in situ reflection high energy electron diffraction (RHEED) and after growth by ex situ X-ray diffraction (XRD). RHEED patterns [Fig. 1(a)], formed by thin, continuous, and contrasted streaks, attest for flat surfaces and the high crystalline quality of the SGA film with [111] as the growth direction. XRD spectra 

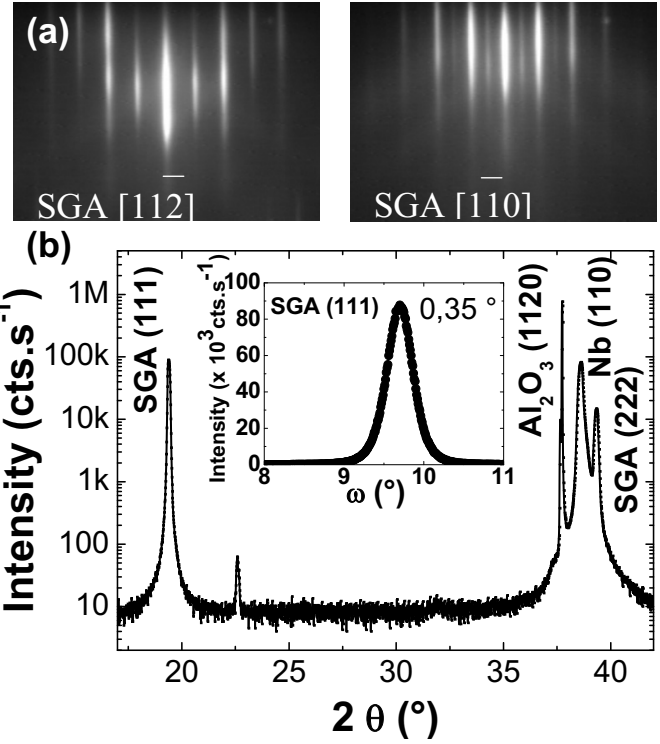

FIG. 1. (a) RHEED patterns collected along two different azimuths after the growth of $1 \mu \mathrm{m}$ (111) SGA (b) $\vartheta / 2 \vartheta$ x-ray scattering measurement using $K \alpha \mathrm{Cu}$ wavelength and rocking curve (inset) measured across the SGA (111) Bragg reflection.

for the SGA layer [Fig. 1(b)] show the (111) diffraction peaks of the expected Laves phase structure with a mosaicity of $0.35^{\circ}$ (see inset).

In order to build up MTJ, the epitaxial SGA layer has to be covered with an insulating layer. This is a crucial issue of our study. Indeed, we have to keep the magnetic properties of the bulk material at the interface. However, interfaces are very sensitive to material mixing that could change the interface SGA stoichiometry or material oxidation. Indeed, insulating material is often composed of oxygen that can lead to a reaction with the electrode material compound. Therefore, we have chosen to cap the SGA layer with a $2 \mathrm{~nm}$ thick Al layer before the exposure to the air for natural oxidation. Compared to other oxidation methods, in pure oxygen ${ }^{16}$ or using oxygen plasma,${ }^{17}$ the oxidation in air is a rather uncontrolled process. Nevertheless, TMR above $10 \%$ have been reported at room temperature in previous studies. ${ }^{18-21}$ RHEED observations performed during and after $\mathrm{Al}$ deposit attest for epitaxial growth of (111) Al with the same hexagonal symmetry as that observed for (111) SGA. The presence of continuous and contrasted streaks also proves the formation of a high quality and flat $\mathrm{Al}$ deposit. After taking the sample out of the MBE chamber, part of the SGA layer covered by $\mathrm{Al}$ was kept so that the behavior of the single SGA layer can be compared to that of the MTJ. The second part of sample was introduced in a sputtering chamber after natural oxidation of the Al layer in air during $12 \mathrm{~h}$. A $[\mathrm{Co}(0.2 \mathrm{~nm}) / \mathrm{Pt}(0.65 \mathrm{~nm})] \times 5$ multilayer was then sputtered to form the counter electrode. This stack has been chosen to ensure perpendicular magnetization. Indeed, (111) SGA films have been shown to exhibit an easy magnetization axis perpendicular to the growth plane, which is attributed to magnetoelastic effects. ${ }^{22}$ The continuous multilayers have been patterned into micron-sized square-shaped MTJs using standard UV lithography and ion etching techniques. This allows perpendicular to film plane tunnel transport measurements in the dc voltage mode using a two-terminal method. (a)

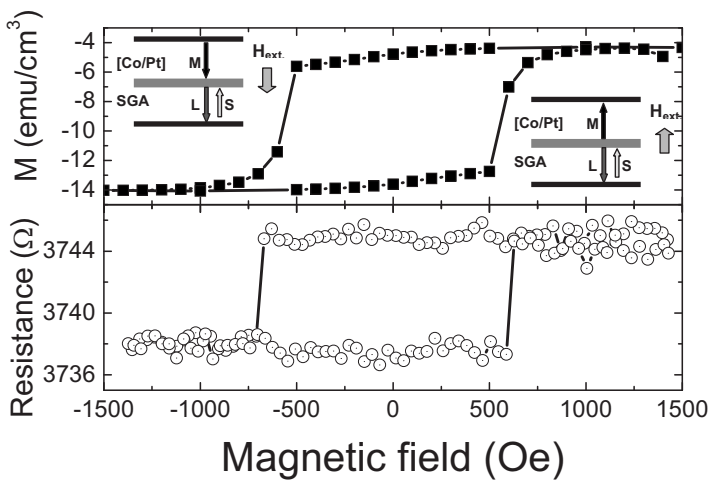

FIG. 2. (a) Minor $\mathrm{M}(\mathrm{H})$ curve and (b) minor $\mathrm{R}(\mathrm{H})$ curve measured at $20 \mathrm{mV}$ and at $15 \mathrm{~K}$ after $1.4 \mathrm{kOe}$ field-cooling on a $\mathrm{SGA} / \mathrm{AlO}_{\mathrm{x}} /[\mathrm{Co} / \mathrm{Pt}]_{5}$ tunnel junction.

Figure 2 shows magnetization and transport measurements performed at low temperature $(15 \mathrm{~K})$ on the $\mathrm{SGA} / \mathrm{AlO}_{\mathrm{x}} /[\mathrm{Co} / \mathrm{Pt}]_{5}$ tunnel junction. Considering the huge increase in the SGA coercive field at low temperature, the $[\mathrm{Co} / \mathrm{Pt}]_{5}$ magnetization reversal is solely achieved in our limited experimental field range. In order to extract qualitative information on the polarization, the SGA magnetization is "initialized" using a field-cooled procedure: just below the Curie temperature, the $\mathrm{S}$ moments are dominant and the positive field-cooling orients the $\mathrm{S}$ moments in the positive direction. This configuration is maintained even below $\mathrm{T}_{\text {comp }}$ despite the dominant $\mathrm{L}$ moments because the cooling field is not strong enough to drive the magnetization reversal. This is obvious on the $\mathrm{M}(\mathrm{H})$ curve at $15 \mathrm{~K}$ [Fig. 2(a)] where the $[\mathrm{Co} / \mathrm{Pt}]_{5}$ hysteresis curve is vertically negatively shifted, and on the $\mathrm{M}(\mathrm{T})$ curve [Fig. 3(a)] where the magnetization is negative below the compensation temperature $(72 \mathrm{~K})$.

The first unambiguous observation is that the $[\mathrm{Co} / \mathrm{Pt}]_{5}$ magnetization reversal is accompanied by an abrupt change in the MTJ resistance [Fig. 2(b)]. Considering the tunnel like variation in resistance with temperature [inset, Fig. 3(b)], ${ }^{23}$ the nonlinear current versus voltage characteristics, the large ratio of the area junction resistance $\left(3.74 \times 10^{5} \Omega \mu \mathrm{m}^{2}\right)$ over sheet resistance of the lead $(0.6 \Omega / \square)$ avoiding current distribution effects, ${ }^{24}$ and the linear variation in the junction resistance with junction surface, this variation in resistance proves the existence of a TMR effect in this SGA based

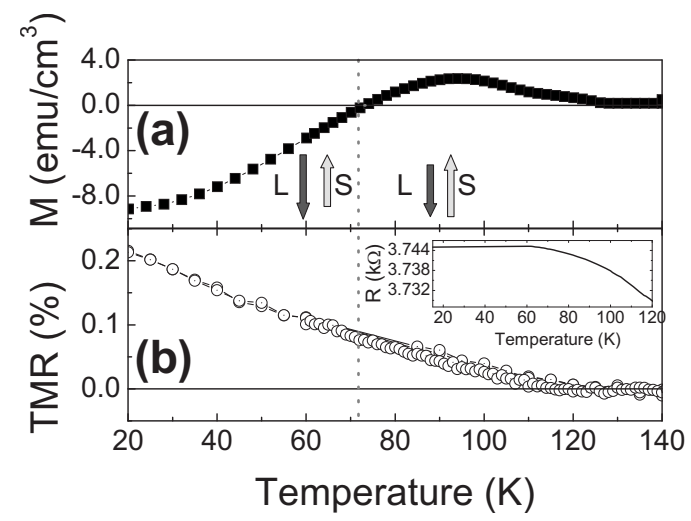

FIG. 3. (a) $\mathrm{M}(\mathrm{T})$ curve measured on the SGA single layer in increasing temperature under zero field after field-cooling under $+70 \mathrm{kOe}$ and (b) $\mathrm{TMR}(\mathrm{T})$ curve measured on a $\mathrm{SGA} / \mathrm{AlO}_{\mathrm{x}} /[\mathrm{Co} / \mathrm{Pt}]_{5}$ tunnel junction at 20 $\mathrm{mV}$. Inset: resistance vs temperature measured at $10 \mathrm{mV}$. 
MTJ. The TMR signal is defined here as $[\mathrm{R}(1.4 \mathrm{kOe})$ $-\mathrm{R}(-1.4 \mathrm{kOe}) / \mathrm{R}(+1.4 \mathrm{kOe})]$.

Contributions from anisotropic MR and anomalous Hall effect are ruled out for both electrodes. Indeed, the SGA magnetization does not switch in the field window used in our study. Moreover the MR effect decreases in increasing temperature [Fig. 3(b)] to vanish in the vicinity of SGA Curie temperature $(125 \mathrm{~K})$ while the $\mathrm{Co} / \mathrm{Pt}$ multilayer remains ferromagnetic well beyond this temperature.

These transport properties, intimately correlated with the $\mathrm{SGA} / \mathrm{AlO}_{\mathrm{x}}$ interface, thus reveal an interface magnetic ordering temperature close to the core of the SGA layer. This is a strong indication of the quality of the $\mathrm{SGA} / \mathrm{AlO}$ interface. Another point of paramount interest is the observation of a finite TMR at the SGA compensation temperature. This constitutes the proof that SGA can simultaneously exhibit zero magnetization and significant polarization of conduction electrons, being thus able to give rise to a spin-polarized current in the magnetic compensated state. One can finally notice the monotonous decrease in the TMR signal versus temperature, without any change in sign at the compensation temperature. A sign inversion, as observed in GdCo-based MTJ, ${ }^{8}$ may have been expected since the dominant magnetic contribution evolves from $\mathrm{L}$ to $\mathrm{S}$ when crossing $\mathrm{T}_{\text {comp }}$. This is not the case in our study because the orientation of SGA magnetic contributions is the same over the entire temperature range, with $\mathrm{S}$ moments always pointing toward positive fields. This highlights that TMR is related to the relative orientation of S contributions, and not of magnetizations, of both electrodes. In this MTJ, the spin parallel (P) state (P alignment of $[\mathrm{Co} / \mathrm{Pt}]$ and SGA S contributions) gives rise to larger resistance than the spin antiparallel (AP) state (AP alignment of $[\mathrm{Co} / \mathrm{Pt}]$ and SGA S contributions) over the entire temperature range. It has been shown that the tunnel polarization of the $\mathrm{Co} / \mathrm{Al}_{2} \mathrm{O}_{3}$ couple is ascribed to the selection of the $s$-character electrons by bonding effects at the $\mathrm{Co}-\mathrm{Al}_{2} \mathrm{O}_{3}$ interface. ${ }^{25}$ This suggested that the positive sign of the Co polarization is associated with the electronic structure at the $\mathrm{Co}-\mathrm{Al}_{2} \mathrm{O}_{3}$ interface rather than with the propagation through the barrier. Thus considering the positive polarization of the Co electrode in our system, the measured larger resistance for the spin P state implies that the tunnel polarization of the SGA electrode should be negative.

This letter reports the proof that SGA can simultaneously exhibit zero magnetization and significant polarization of conduction electrons, being thus able to give rise to a spinpolarized current in the magnetic compensated state. This paves the way to interesting perspectives for spin torque effects.

The work presented in this letter is partly supported by La Région Lorraine. The authors acknowledge S. Suire for technical assistance.

${ }^{1}$ M. Jullière, Phys. Lett. 54A, 225 (1975).

${ }^{2}$ P. Rottländer, M. Hehn, O. Lenoble, and A. Schuhl, Appl. Phys. Lett. 78, 3274 (2001), and references therein.

${ }^{3}$ J. Faure-Vincent, C. Tiusan, E. Jouguelet, F. Canet, M. Sajieddine, C. Bellouard, E. Popova, M. Hehn, F. Montaigne, and A. Schuhl, Appl. Phys. Lett. 82, 4507 (2003), and references therein.

${ }^{4}$ S. Ikeda, K. Miura, H. Yamamoto, K. Mizunuma, H. D. Gan, M. Endo, S. Kanai, J. Hayakawa, F. Matsukura, and H. Ohno, Nature Mater. 322, 996 (2010).

${ }^{5}$ D. Ebke, P. Thomas, O. Schebaum, M. Schäfers, D. Nissen, V. Drewello, A. Hütten, and A. Thomas, J. Magn. Magn. Mater. 322, 996 (2010).

${ }^{6}$ H. van Leuken and R. A. de Groot, Phys. Rev. Lett. 74, 1171 (1995).

${ }^{7}$ K. Özdogan, I. Galanakis, E. Sasioglu, and B. Aktas, J. Phys.: Condens. Matter 18, 2905 (2006).

${ }^{8}$ C. Kaiser, A. F. Panchula, and S. S. P. Parkin, Phys. Rev. Lett. 95, 047202 (2005).

${ }^{9}$ H. Adachi and H. Ino, Nature (London) 401, 148 (1999).

${ }^{10}$ J. W. Taylor, J. A. Duffy, A. M. Bebb, M. R. Lees, L. Bouchenoire, S. D. Brown, and M. J. Cooper, Phys. Rev. B 66, 161319 (2002).

${ }^{11}$ H. Adachi, H. Kawata, H. Hashimoto, Y. Sato, I. Matsumoto, and Y. Tanaka, Phys. Rev. Lett. 87, 127202 (2001).

${ }^{12}$ S. Qiao, A. Kimura, H. Adachi, K. Iori, K. Miyamoto, T. Xie, H. Namatame, M. Taniguchi, A. Tanaka, T. Muro, S. Imada, and S. Suga, Phys. Rev. B 70, 134418 (2004).

${ }^{13}$ A. Avisou, C. Dufour, K. Dumesnil, and D. Pierre, J. Cryst. Growth 297, 239 (2006).

${ }^{14}$ A. Avisou, K. Dumesnil, and C. Dufour, J. Magn. Magn. Mater. 316, 317 (2007).

${ }^{15}$ A. Avisou, C. Dufour, K. Dumesnil, A. Rogalev, F. Wilhelm, and E. Snoeck, J. Phys.: Condens. Matter 20, 265001 (2008).

${ }^{16}$ H. Tsuge and T. Mitsuzuka, Appl. Phys. Lett. 71, 3296 (1997).

${ }^{17}$ J. Nassar, M. Hehn, A. Vaurès, F. Petroff, and A. Fert, Appl. Phys. Lett. 73, 698 (1998).

${ }^{18}$ T. Miyazaki and N. Tezuka, J. Magn. Magn. Mater. 139, L231 (1995).

${ }^{19}$ H. Chen, Q. Y. Xu, G. Ni, J. Lu, H. Sang, S. Y. Zhang, and Y. W. Du, J. Appl. Phys. 85, 5798 (1999).

${ }^{20}$ S. Kumagai, N. Tezuka, and T. Miyazaki, Jpn. J. Appl. Phys., Part 2 36, L1498 (1997).

${ }^{21}$ M. Sato and K. Kobayashi, IEEE Trans. Magn. 33, 3553 (1997).

${ }^{22}$ A. Avisou, C. Dufour, and K. Dumesnil, J. Appl. Phys. 103, $07 E 135$ (2008).

${ }^{23}$ J. J. Åkerman, R. Escudero, C. Leighton, S. Kim, D. A. Rabson, R. W. Dave, J. M. Slaughter, and I. K. Schuller, J. Magn. Magn. Mater. 240, 86 (2002)

${ }^{24}$ F. Montaigne, F. Nguyen Van Dau, and A. Schuhl, J. Magn. Magn. Mater. 217, 231 (2000).

${ }^{25}$ J. M. De Teresa, A. Barthelemy, A. Fert, J. P. Contour, F. Montaigne, and P. Seneor, Science 286, 507 (1999). 